\title{
Drain after elective laparoscopic cholecystectomy. A randomized multicentre controlled trial
}

\author{
Marcello Picchio - Francesco De Angelis • Settimio Zazza • \\ Annalisa Di Filippo • Raffaello Mancini - Giada Pattaro • \\ Francesco Stipa • Adewale Oluseye Adisa • Giuseppe Marino • \\ Erasmo Spaziani
}

Received: 15 July 2011/Accepted: 13 March 2012/Published online: 27 April 2012

(C) The Author(s) 2012. This article is published with open access at Springerlink.com

\begin{abstract}
Background Routine drainage after laparoscopic cholecystectomy is still debatable. The present study was designed to assess the role of drains in laparoscopic cholecystectomy performed for nonacutely inflamed gallbladder. Methods After laparoscopic gallbladder removal, 53 patients were randomized to have a suction drain positioned in the subhepatic space and 53 patients to have a sham drain. The primary outcome measure was the presence of subhepatic fluid collection at abdominal ultrasonography, performed $24 \mathrm{~h}$ after surgery. Secondary outcome measures were postoperative abdominal and shoulder tip pain, use of analgesics, nausea, vomiting, and morbidity.

Results Subhepatic fluid collection was not found in 45 patients (84.9\%) in group A and in 46 patients $(86.8 \%)$ in
\end{abstract}

M. Picchio · S. Zazza · G. Marino

Department of Surgery, Hospital "P. Colombo", Via Orti

Ginnetti 7, 00049 Velletri, Rome, Italy

M. Picchio $(\bowtie)$

Department of Surgery, Hospital "P. Colombo", Via Giulio

Cesare, 58, 04100 Latina, Italy

e-mail: marcellopicchio@libero.it

F. De Angelis · A. Di Filippo - R. Mancini - G. Pattaro .

E. Spaziani

Department of Surgery, University of Rome "La Sapienza",

Polo Pontino, Via Firenze, 04019 Terracina, Latina, Italy

F. Stipa

Department of Surgery, Hospital "S. Giovanni-Addolorata", Via dell'Amba Aradam 9, 00184 Rome, Italy

A. O. Adisa

Department of Surgery, Obafemi Awolowo University, Ile-Ife 220005, Nigeria group B (difference 1.9 (95\% confidence interval - 11.37 to $15.17 ; P=0.998$ ). No significant difference in visual analogue scale scores with respect to abdominal and shoulder pain, use of parenteral ketorolac, nausea, and vomiting were found in either group. Two (1.9\%) significant hemorrhagic events occurred postoperatively. Wound infection was observed in three patients $(5.7 \%)$ in group A and two patients $(3.8 \%)$ in group B (difference 1.9 (95\% CI -6.19 to $9.99 ; P=0.997)$.

Conclusions The present study was unable to prove that the drain was useful in elective, uncomplicated LC.

Keywords Cholecystectomy - Laparoscopy · Drainage

Laparoscopic cholecystectomy (LC) is the current preferred method of cholecystectomy. The role of routine drainage after LC to decrease postoperative morbidity is still an issue of considerable debate. In a recent Australian survey, surgeons were evenly divided into those who used drains routinely, those who always drained, and those who never drained after LC [1]. The main reason to use drains in laparoscopic cholecystectomy is to avoid bile and blood collection requiring subsequent open procedures. However, a Cochrane Database Systematic Review found no evidence to support the use of drains in laparoscopic cholecystectomy [2]. The limitations of this review include the few randomized, clinical trials with high methodological quality and heterogeneity in the measurement of outcomes. The goal of the present multicentre trial was to assess the role of drains in LC, performed for nonacutely inflamed gallbladder. In particular, the efficacy of drain in preventing postoperative abdominal fluid collections and improving surgery outcome was evaluated. 


\section{Materials and methods}

From December 1, 2009 to December 31, 2010, 270 patients aged 18 years and older were submitted to elective LC at the three participating hospitals (Hospital "P. Colombo," Velletri, Italy; University of Rome "La Sapienza", Polo Pontino, Terracina, Italy; Obafemi Awolowo University, Ile-Ife, Nigeria). Patients with acute cholecystitis, cholangitis, or pancreatitis were not included. If intraoperative common bile duct exploration or any other additional procedure were performed, patients also were excluded. Patients with evidence of concomitant choledocholithiasis were treated with preoperative endoscopic retrograde cholangiopancreatography and common bile duct clearance. After approval by local bioethics committees, informed consent was obtained preoperatively on hospital admission. The following clinical data were evaluated: age, sex, body mass index (BMI), and American Association of Anesthesiologists (ASA) risk.

\section{Surgical management}

All operations were performed by surgeons with a previous minimum experience of $50 \mathrm{LC}$. Under general anesthesia, the abdomen was insufflated with $\mathrm{CO}_{2}$ after the introduction of the first $10-\mathrm{mm}$ trocar with the Hasson technique through an infraumbilical incision. The other $10-\mathrm{mm}$ and two 5-mm trocars were inserted through appropriate subxiphoid, subcostal midclavicular, and subcostal anterior axillary incisions. The pneumoperitoneum pressure and $\mathrm{CO}_{2}$ flow rate were set at $10 \mathrm{mmHg}$ and $2 \mathrm{~L} / \mathrm{min}$, respectively. A standard retrograde cholecystectomy with previous isolation and section between 10-mm clips of cystic duct and artery was always performed. The gallbladder was always bagged and retrieved through the umbilical port. The duration of the operation (from infraumbilical skin incision to pulling off the trocars), bile spillage, and additional complications also were recorded.

\section{Randomization}

After gallbladder removal with containing bag, the patients, who had no serious intraoperative complications, such as significant biliary and/or vascular injury or bleeding $(>100 \mathrm{~mL})$, were randomly allocated to undergo the placement of a drain in the subhepatic space (group A) or a sham drain (group B). Randomization was computergenerated, using numbered and sealed envelopes, which were opened in the operating room at the end of surgery before drain fixation to the skin. The polyethylene, 5.7$\mathrm{mm}$, multiparous, tube drain was threaded through the most lateral 5-mm trocar. In group B, after the surgeon inserted the drain, a nurse of the operatory room pulled out the drain outside the port, shortened the tube, and fixed the end to the skin with a tape after blocking the tip with a bead. All drains in both groups were connected to a $500-\mathrm{mL}$ closed suction reservoir. This way, the operator, the patients, and the assessors were blinded to the intervention.

\section{Postoperative monitoring}

Patients were given a standard deep vein thrombosis prophylaxis. Postoperative pain was evaluated as follows: (1) parenteral diclofenac requirements were recorded after the patient was instructed to ask for pain relief liberally; (2) a visual analogue scale (VAS) [3] from 0 (no pain) to 10 (worst pain imaginable) was completed by each patient $24 \mathrm{~h}$ after surgery and at least $2 \mathrm{~h}$ after any eventual diclofenac assumption with respect to either abdominal and shoulder pain. An abdominal ultrasonography was routinely performed on the first postoperative day with the goal to detect any fluid collection. If present, the volume of subhepatic collection was calculated. Ultrasound examinations were performed using an Aloka Prosound Alpha $10^{\circledR}$ with a $1.5-\mathrm{MHz}$, convex probe by experienced radiologists.

The drain was removed $24 \mathrm{~h}$ after surgery, unless there was bile (any amount) or $100 \mathrm{~mL}$ of blood in the drain bag. In case the drain had to stay in place for bile leak, it was not removed, unless the leak had completely ceased. In case the drain had to stay in place for bleeding, it was removed when the amount was $100 \mathrm{~mL} / 24 \mathrm{~h}$ and the patient was hemodynamically stable with stable hemoglobin (no decrease $>1 \mathrm{~g} / \mathrm{dL}$ ). Patients were discharged on the second postoperative day, unless the drain had to stay in place for any of the reasons mentioned and/or intraabdominal fluid collection $>50 \mathrm{~mL}$ was detected at ultrasonographic examination and no other complications had occurred. Intra-abdominal fluid collections $>50 \mathrm{~mL}$ were followed up with serial ultrasonographic examinations and patients were discharged if no increase was detected.

Postoperative problems and complications were recorded within 4 weeks after operation. Patients were reviewed at 1 week and 4 weeks postoperatively. An upper abdomen ultrasonography was routinely performed 1 week after surgery. Outcome assessors were unaware of patients' allocation.

\section{Statistical analysis}

The primary outcome measure was the presence of subhepatic fluid collection at ultrasonographic examination of the abdomen $24 \mathrm{~h}$ after surgery. Secondary outcome measures were postoperative abdominal and shoulder tip pain, use of analgesics, nausea, vomiting, and morbidity.

Sample size calculation was based on the goal of detecting a difference of $20 \%$ in the proportion of patients 
with absence of subhepatic fluid collection at postoperative ultrasonography, assuming from a previous personal series of 40 patients, submitted to elective laparoscopic cholecystectomy with drainage, that $78 \%$ of cases showed no subhepatic collection at ultrasonography. With a type I error of 0.05 and a type II error of 0.10 for a two-tailed test, 53 patients per group were required.

Pearson $\chi^{2}$ test was used for categorical data. A $95 \%$ confidence interval (CI) on the difference in proportions was calculated. The Mann-Whitney $U$ test was used to compare not normally distributed samples. All tests were two-tailed, and the level of significance was 0.05. All data were compiled by an independent participant unaware of patients' allocation, and the results were analyzed using Medcalc ${ }^{\circledR}$ version 7.3 (Frank Schoonjanas, Broekstraat, Belgium).

\section{Results}

The profile of the trial is shown in Fig. 1. The number of patients who entered the study in each participating center was as follows: 39 patients in the Hospital "P. Colombo," Velletri, Italy; 35 patients in the University of Rome "La Sapienza"- Polo Pontino, Terracina, Italy; 32 patients in the Obafemi Awolowo University, Ile-Ife, Nigeria). No violation of the protocol was registered. Both groups were comparable with respect to sex, age, BMI, ASA, intraoperative spillage, mean operative time, and median postoperative hospital stay (Table 1). No significant intraoperative morbidity occurred.

Abdominal ultrasonography did not show any subhepatic fluid collection in 45 patients $(84.9 \%)$ in group A and in 46 patients $(86.8 \%$ ) in group B (difference 1.9 (95\% CI, -11.37 to $15.17 ; P=0.998)$. If present, median $(95 \% \mathrm{CI})$ subhepatic collection was $30 \mathrm{~mL}(20-40 \mathrm{~mL})$ in group A and $30 \mathrm{~mL}(20-40 \mathrm{~mL})$ in group $\mathrm{B}(P=0.779$; Mann-Whitney $U$ test). All subhepatic collections disappeared at ultrasonographic examination of the abdomen, performed 1 week after surgery. Median (95\% CI) abdominal pain scores $24 \mathrm{~h}$ after operation was $3(2-4)$ in group $\mathrm{A}$ and 2 (2-3) in group B $(P=0.201$; MannWhitney $U$ test). Median $(95 \% \mathrm{CI})$ shoulder pain scores $24 \mathrm{~h}$ after operation was $0(0-1)$ in group $\mathrm{A}$ and $0(0-0)$ in group $\mathrm{B}(P=0.324$; Mann-Whitney $U$ test $)$. Median (95\% CI) parenteral ketorolac consumed was $60 \mathrm{~mL}$ $(30-60 \mathrm{~mL})$ in group A and $30 \mathrm{~mL}(30-30 \mathrm{~mL})$ in group B $(P=0.126$; Mann-Whitney $U$ test $)$. Four patients $(7.5 \%)$ in group A and three patients (5.8\%) in group B suffered from nausea $24 \mathrm{~h}$ after operation (difference $1.7 \%(95 \%$ CI, -7.78 to 11.18$) ; P=0.969)$. One patient $(1.9 \%)$ in

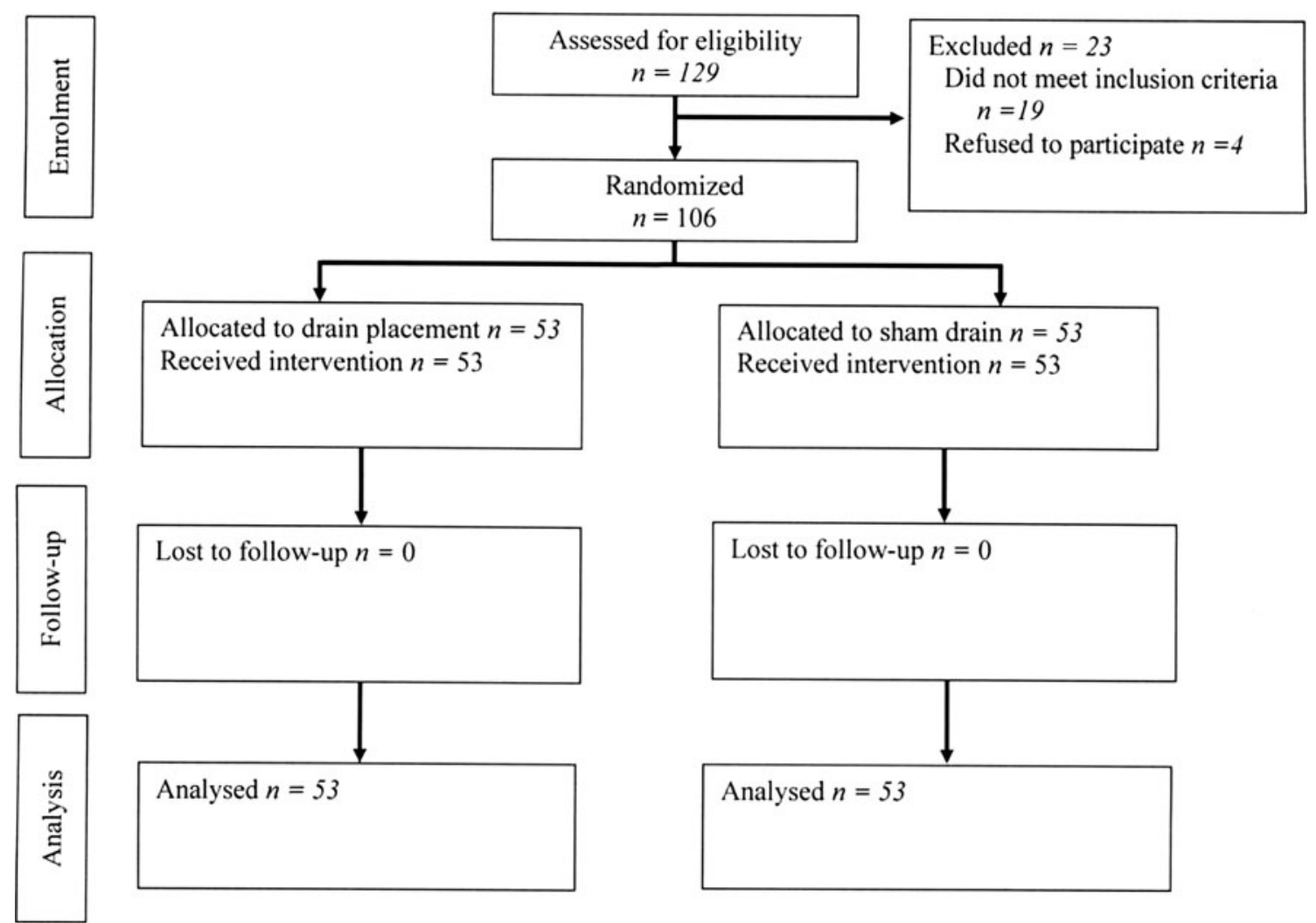

Fig. 1 CONSORT flow-chart for the randomized study 
Table 1 Characteristics of patients

\begin{tabular}{lll}
\hline Characteristic & Group A $(n=53)$ & Group B $(n=53)$ \\
\hline Gallbladder disease & & \\
Cholelithiasis & $49(92.5)$ & $49(92.5)$ \\
Gallbladder polyp & $4(7.5)$ & $4(7.5)$ \\
Sex & & \\
M & $11(20.8)$ & $13(24.5)$ \\
F & $42(79.2)$ & $40(75.5)$ \\
Mean age $(95 \%$ CI) (year) & $48.6(44.7-52.5)$ & $47.1(42.4-51.8)$ \\
BMI (95\% CI) & $26.7(25.5-27.9)$ & $24.7(23.7-25.7)$ \\
ASA & & $22(41.5)$ \\
I & $27(50.9)$ & $19(35.8)$ \\
II & $16(30.2)$ & $12(22.6)$ \\
III & $10(18.9)$ & $60.7(55.5-65.9)$ \\
Operative mean time (95 \% CI) (min) & $67.1(62.8-71.3)$ & $2(3.8)$ \\
Intraoperative bile spillage & $2(3.8)$ & $2(2-10)$ \\
Median (range) postoperative hospital stay (days) & $2(2-7)$ & \\
\hline
\end{tabular}

group A and two patients $(3.8 \%)$ in group B suffered from vomiting [difference $1.9 \%(95 \% \mathrm{CI},-4.43$ to 8.23$)$; $P=0.997]$.

Two (1.9\%) significant postoperative complications occurred. One patient in group A presented a 200-mL collection in the drain with hemodynamic instability $4 \mathrm{~h}$ after surgery, requiring emergency laparotomy with hemostasis of an iatrogenic lesion of the gallbladder fossa. The other patient in group B developed abrupt and intense abdominal pain with tachycardia in the second postoperative day. Ultrasonography and computed tomography scan of the abdomen showed the presence of a subcapsular hepatic hematoma involving the entire periphery of the right lobe. The patient was treated conservatively and serial ultrasonographic and tomographic examinations of the abdomen showed the progressive reduction of the subcapsular hematoma, which disappeared 5 months after surgery. Infraumbilical port-site infection occurred in five patients $(4.7 \%)$ in the entire study group. Wound infection occurred in three patients $(5.7 \%)$ in group A and two patients $(3.8 \%)$ in group B [difference $1.9(95 \% \mathrm{CI}$, -6.19 to $9.99 ; P=0.997]$.

\section{Discussion}

Cholecystectomy is the second most common operation in gastrointestinal surgery after appendectomy. However, there are still limited data on the value of prophylactic drains for LC. The recent Cochrane Database Systematic Review [2] only found two studies with high methodological quality $[4,5]$. Since then, only one randomized trial was published with a large number of patients enrolled and adequate methodology [6]. The present study represents a rare instance in surgery where an adequate blinding was performed.

Traditionally, drains were used for the early detection of bile leaks and any unsuspected hemorrhage and to evacuate abdominal fluid collections without the need for more invasive procedures. At present, the rate of biliary complications after LC is $0.4 \%$ (range, 0.1-0.9\%) [7]. Postoperative hemorrhagic complications are very rare. Given the low proportion of these complications in patients submitted to elective laparoscopic cholecystectomy, it is unlikely that any trial will be powered to measure differences in these specific complications. In the present study, significant postoperative complications were rare. In particular, no postoperative bile leak was documented. Two postoperative hemorrhagic complications occurred. In one patient, the drain evidenced a significant hemorrhage. The absence of subhepatic fluid collections after cholecystectomy is strongly associated with an uncomplicated postoperative recovery [8]. The efficacy of drains to evacuate subhepatic collections may justify their use to prevent postoperative complications. However, experimental studies [9] showed that, when a drain is inserted in the peritoneal cavity that contains no fluids, it is quickly surrounded by omentum and completely occluded within $48 \mathrm{~h}$. The present study was unable to prove that the drain has any influence on the presence and severity of subhepatic fluid collection after LC. Drains are supposed to be much more efficient in draining bile than other types of intra-abdominal collections. However, large series from the era of open cholecystectomy showed that most patients, who underwent laparotomy for postcholecystectomy bile peritonitis, had drains placed, suggesting that drain placement does not detect this complication effectively [10-12]. Drains also 
are not effective to treat bile leak or bleeding in elective LC [6]. Moreover, a recent study showed that drains increase the occurrence of fluid in the subhepatic space after LC [13]. Possible causes are irritation from the foreign material of the drain, prevention of tissue tamponade, creation of dead space, and the effects of vacuum suction from the drain. Ultrasonographic studies clearly demonstrated that most postcholecystectomy collections remain asymptomatic and are absorbed by the peritoneum $[14,15]$. In the present trial, all subhepatic collections, evidenced on the first postoperative day, were absent at 1-week control ultrasonography.

Port-site infection is a minor complication that affects $1.1-7.9 \%$ of patients after LC $[16,17]$. The use of drains seems to improve the incidence of this complication, possibly related to the presence of a foreign body [6]. However, morbidity was not increased if short-term drains were used in open cholecystectomy. Williams et al. [18] showed an increase in postoperative morbidity when Penrose drains were left longer than $48 \mathrm{~h}$. In this study, the drain was routinely removed on the first postoperative day. The short permanence of the drain may account for the lack of increase in wound infections associated with the presence of a subhepatic drain. All wound infections were located at the level of the infraumbilical incision in our series. Antibiotic prophylaxis does not reduce the rate of umbilical wound infection with respect to bag extraction of the gallbladder [17]. Topical antibiotics were found to be effective in reducing this bothering complication [19].

The effect of subhepatic drain on postoperative pain is controversial. Significant reduction of postoperative pain in patient without drain insertion with respect to those with subhepatic drains was reported in the trial of Tzovaras et al. [6]. On the contrary, the study of Hawasli et al. [20] failed to find any difference. Jorgensen et al. [21] showed that the use of a suction drain in LC decreases shoulder pain by allowing carbon dioxide gas to escape with respect to passive drain. That is the reason why we chose to position a suction drain in group A. Our data were unable to prove that suction drain has any effect on either abdominal or shoulder tip pain after LC.

Postoperative nausea and vomiting has been reported with an incidence of 53-72\% after laparoscopic cholecystectomy [22]. However its incidence tends to decrease during the early postoperative recovery [23, 24]. Our data showed a low incidence of postoperative nausea and vomiting assessed $24 \mathrm{~h}$ after operation. The presence of drain did not influence its incidence.

In conclusion, the present study was unable to prove that the drain was useful in elective, uncomplicated LC without acute cholecystitis, cholangitis, or pancreatitis and no significant intraoperative morbidity.
Acknowledgments The authors thank Dr. Jacopo Martellucci for revising the final draft of the manuscript, and Dr. Ilaria Tanga, Dr. Giuseppe Pietricola, and Dr. Piero Francioni for the protocol of ultrasonographic examinations used in the study.

Contributors The following surgeons performed the operations: Ettore Greco, Giuseppe Marino, Claudio Mascetti, Andrea Muggianu, Maurizio Ottaviani, Marcello Picchio, Settimio Zazza (Department of Surgery, Hospital "P.Colombo", Velletri, Italy); Raffaello Mancini, Erasmo Spaziani (Department of Surgery, University of Rome "La Sapienza"- Polo Pontino, Terracina, Italy); Adewale Oluseye Adisa (Department of Surgery, Obafemi Awolowo University, Ile-Ife, Nigeria).

Disclosures Marcello Picchio, Francesco De Angelis, Settimio Zazza, Annalisa Di Filippo, Raffaello Mancini, Giada Pattaro, Francesco Stipa, Adewale Oluseye Adisa, Giuseppe Marino, and Erasmo Spaziani have no conflict of interest or financial ties to disclose.

Open Access This article is distributed under the terms of the Creative Commons Attribution License which permits any use, distribution, and reproduction in any medium, provided the original author(s) and the source are credited.

\section{References}

1. Askew J (2005) A survey of the current surgical treatment of gallstones in Queensland. ANZ J Surg 75:1086-1089

2. Gurusamy KS, Samraj K, Mullerat P, Davidson BR (2007) Routine abdominal drainage for uncomplicated laparoscopic cholecystectomy. Cochrane Database Syst Rev 3:CD006004

3. Huskisson EC (1974) Measurement of pain. Lancet 2:1127

4. Nomdedeu J, Escrig J, Salvador JL (1996) Systematic placement of drains in laparoscopic cholecystectomy. A prospective study. Revista de la Sociedad Valenciana de Patologia Digestiva 15: 299-300

5. Capitanich P, Segundo UL, Malizia P, Herrera J, Iovaldi ML (2005) Usefulness of prophylactic drainage in laparoscopic cholecystectomy. Randomized prospective report. Prensa Med Argent 92:623-627

6. Tzovaras G, Liakou P, Fafoulakis F, Baloyiannis I, Zacharoulis D, Hatzitheofilou C (2009) Is there a role for drain use in elective laparoscopic cholecystectomy? A controlled randomized trial. Am J Surg 197:759-763

7. Chiche L, Letoublon C (2010) Traitment des complications de la cholécystctomie. In: EMC, Techniques chirurgicales-Appareil digestif, pp. 40-960. Elsevier Masson SAS, Paris

8. Håkansson K, Leander P, Ekberg O, Håkansson HO (2001) MR imaging of upper abdomen following cholecystectomy. Normal and abnormal findings. Acta Radiol 42:181-186

9. Agrama HM, Blackwood JM, Brown CS, Machiedo GW, Rush BF (1976) Functional longevity of intraperitoneal drains: an experimental evaluation. Am J Surg 132:418-421

10. Monson RTJ, Keane BVF, Brenman GT (1991) Cholecystectomy is safer without drainage: the results of a prospective, randomised clinical trial. Surgery 109:740-746

11. Farha JG, Chang CF, Mathews HE (1981) Drainage in elective cholecystectomy. Am J Surg 142:678-680

12. Budd CD, Cochran CR, Fouty JW (1982) Cholecystectomy with and without drainage. Am J Surg 143:307-309

13. Georgiou C, Demetriou N, Pallaris T, Theodosopoulos T, Katsouyanni K, Polymeneas G (2011) Is the routine use of drainage 
after elective laparoscopic cholecystectomy justified? A randomized trial. J Laparoendosc Adv Surg Tech A 21:119-123

14. Wright NB, Williamson VC (1994) Ultrasound findings following laparoscopic cholecystectomy. Br J Radiol 67:429-430

15. Farrell TA, Geraghty JG, Keeling F (1993) Abdominal ultrasonography following laparoscopic cholecystectomy: a prospective study. Clin Radiol 47:111-113

16. The Southern Surgeons Club (1991) A prospective analysis of 1518 laparoscopic cholecystectomies. N Engl J Med 324: 1073-1078

17. Harling R, Moorjani N, Perry C, MacGowan AP, Thompson MH (2000) A prospective, randomised trial of prophylactic antibiotics versus bag extraction in the prophylaxis of wound infection in laparoscopic cholecystectomy. Ann R Coll Surg Engl 82:408-410

18. Williams CB, Halpin DS, Knox AJ (1972) Drainage following cholecystectomy. Br J Surg 59:293-296

19. Neri V, Fersini A, Ambrosi A, Tartaglia N, Valentino TP (2008) Umbilical port-site complications in laparoscopic cholecystectomy: role of topical antibiotic therapy. JSLS 12:126-132
20. Hawasli A, Brown E (1994) The effect of drains in laparoscopic cholecystectomy. J Laparoendoscopic Surg 4:393-398

21. Jorgensen JO, Gillies RB, Hunt DR (1995) A simple and effective way to reduce postoperative pain after laparoscopic cholecystectomy. ANZ J Surg 65:466-469

22. Feo CV, Sortini D, Ragazzi R, De Palma M, Liboni A (2006) Randomized clinical trial of the effect of preoperative dexamethasone on nausea and vomiting after laparoscopic cholecystectomy. Br J Surg 93:295-299

23. Nursal TZ, Yildirim S, Tarim A, Noyan T, Poyraz P, Tuna N, Haberal M (2003) Effect of drainage on postoperative nausea, vomiting, and pain after laparoscopic cholecystectomy. Langenbecks Arch Surg 388:95-100

24. Sánchez-Rodríguez PE, Fuentes-Orozco C, González-Ojeda A (2010) Effect of dexamethasone on postoperative symptoms in patients undergoing elective laparoscopic cholecystectomy: randomized clinical trial. World J Surg 34:895-900 